\title{
Neural Networks for Improved Target Differentiation with Sonar
}

\author{
Naima Ait Oufroukh and Etienne Colle \\ Laboratoire Systèmes Complexes, 40, rue du Pelvoux \\ 91020 Evry Cedex France \\ \{Aitoufroukh.N, ecolle\}@iup.univ-evry.fr
}

\begin{abstract}
This study investigates the processing of sonar signals with neural networks for robust recognition of indoor robot environment composed of simple objects (plane, corner, edge and cylinder). The neural networks can differentiate more targets with higher accuracy. It achieves this by exploiting the identifying features extracted from sonar signals. In this paper we compare two different architectures of neural networks (global and specialized structure) in term of classification rates, the best classifier obtained is used to recognize a robot environment. The results strengthen our claims that sonar can be used as a viable system for object recognition in robotics and other application domains.
\end{abstract}

\section{Introduction}

In contrast, typical robotics applications only use sonar as a range finder, measuring the time-of-flight of the leading edge of the ultrasonic echo to determine the distance of the object that generated the echo [1,2]. Target differentiation is of importance for intelligent systems that need to interact with and autonomously operate in their environment. Many works have studied ultrasonic sensors for this purpose [3,4]. Sonar is a very useful and cost-effective mode of sensing for mobile robots. The fact that sonar sensors are light, robust and inexpensive devices has led to their widespread use in applications such as map building [5], target tracking [6], and obstacle avoidance [7]. Although there are difficulties in the interpretation of sonar data due to poor angular resolution of sonar, multiple reflections and establishing correspondence between multiple echoes on different receivers [4], these difficulties can be overcome by employing an intelligent processing on sonar signals. This paper investigates the use of neural networks to process sonar signals encountered in target differentiation application for indoor environments. The motivation behind the use of neural network classifiers in sonar or radar systems is the desire to emulate the remarkable perception and pattern recognition capabilities of humans and animals [8], Carpenter used a Fuzzy ARTMAP neural network [9] to classify echoes from five objects. A comparison between neural networks and standard classifiers for radar-specific emitter identification is provided by Willson [10]. Neural networks have also been used in the classification of sonar returns from undersea targets [8]. The purpose of this paper is to recognize a real indoor environment by using intelligent processing and ultrasonic 
sensors. Before classification, we determine the most discriminant features set with several methods: sequential methods (Backward and Forward), optimal method (Branch and bound).

The classifier used is determined by a comparison of two neural networks. The networks considered are the Global Neural Network (GNN) and the Specialized Neural Networks (SNN).

\section{Sonar System Description}

The system we presented uses two readily available 6500-series Polaroid sonar with $80 \mathrm{~mm}$ between centers. The sensors are mounted on the step by step motor (the step $=1.8^{\circ}$ ). It is contained in the copper box in order to avoid the interference between magnetic field and sonar signal. The sensors are employed as a transmitter/receiver $(\mathrm{T} / \mathrm{R})$ and a receiver $(\mathrm{R})$. They are characterized by a $10 \mathrm{~m}$ range and a beam aperture $\theta_{0}$ of about $\theta_{0}=\sin ^{-1}(0.61 \lambda / a)$. Where $\lambda=c / f_{0}$ is the wavelength of the acoustic signal, $a=14 \mathrm{~mm}$ is the radius of the transducer and $f_{0}=50 \mathrm{kHz}$ is the resonance frequency of the sensor. The most common sonar ranging system is based on the time-of-flight (TOF) which is the time elapsed between the transmission and the reception of a pulse. In commonly used TOF systems, an echo is produced when the transmitted pulse encounters an object and a range value $r=c t_{0} / 2$ is produced with simple thresholding [5]. Here $t_{0}$ is TOF and $c$ is the speed of sound in air $(c=343,3$ $\mathrm{m} / \mathrm{sec})$.

\section{Target Differentiation}

The target primitives modeled in this study are plane, corner, edge and cylinder with different dimension (Figure 1). Since the wavelength $(\lambda=6.8 \mathrm{~mm})$ is much larger than the typical roughness of used object surfaces, in this case, targets reflect acoustic beams specularly, like a mirror [5].
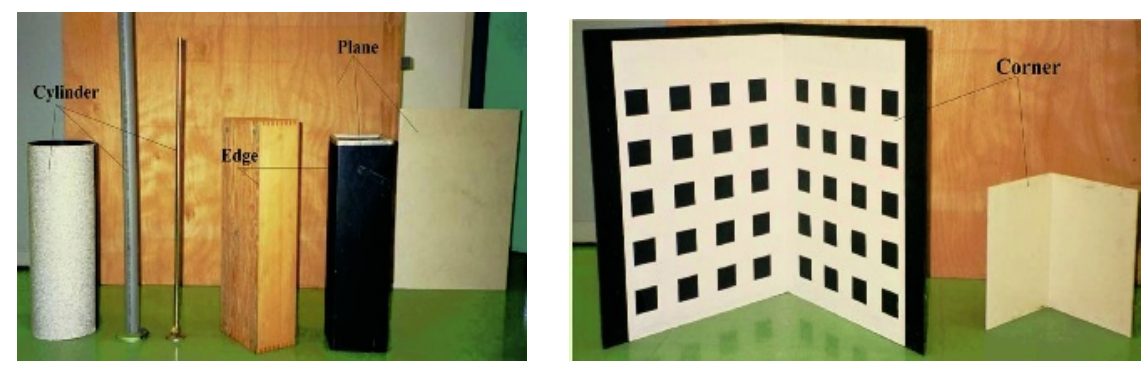

Fig. 1. Targets differentiated in this study. 


\section{Feature Vector}

\subsection{Dimensionality Reduction}

There are two main reasons to keep the dimensionality of the pattern representation (i.e., the number of features) as small as possible: measurement cost and classification accuracy. A limited yet salient feature set simplifies both the pattern representation and the classifiers that are built on the selected representation. Consequently, the resulting classifier will be faster and will use less memory. Moreover, as stated earlier, a small number of features can alleviate the curse of dimensionality when the number of training samples is limited. On the other hand, a reduction of the number of features may lead to a loss in the discrimination power and thereby decreases the accuracy of the resulting recognition system. The classification depends strongly on the features selected to represent the sample. Feature extraction is achieved by an heuristic method. Good feature selection method is an essential step in a classification system [11], which permits to reduce the space of class representation. Among the existing criteria we use the following criteria:

$$
J=\operatorname{trace}\left(\hat{\Sigma}_{W}^{-1} \hat{\Sigma}_{b}\right)
$$

Where $\hat{\Sigma}_{\text {w }}$ is the estimated intra group covariance matrix, which characterizes the dispersion of points in a class. $\hat{\Sigma}_{\mathrm{b}}$ is the estimated inter group covariance matrix which characterizes the dispersion of classes between them.

We made comparison of two feature selection algorithms, with the rate of classification. Forward and Backward sequential selection (FSS, BSS) [12,13] are the most common sequential search algorithms. FSS begins with zero features, evaluates all subsets with exactly one feature and selects the one with largest criteria. BSS instead begins with all features and repeatedly removes a feature whose removal causes the least decrease of criteria. At the k step, we add or remove the feature $\xi_{j}$ as:

$$
J\left(\Xi_{k} \pm \xi_{j}\right) \geq J\left(\Xi_{k} \pm \xi_{i}\right) \quad \mathrm{i}=1, \mathrm{~d}-\mathrm{k} \quad \mathrm{i} \neq \mathrm{j}
$$

Where $\mathrm{J}$ is the criteria (3), $\Xi_{k}$ is the whole set of features minus $\mathrm{k}$ features. The remaining parameters are most discriminative.

After a rough selection of parameters we apply the branch and bound method (BAB) to refine the result. This method examines all feature subsets. It will always find an optimal solution but with cost of computational time. We look for $\Xi^{*}$ as:

$$
\left\{\begin{array}{l}
\Xi \subset \Xi^{*}=\left\{\xi^{*}, \ldots, \xi^{*}\right\} \\
J\left(\Xi^{*}\right)=\operatorname{Max}\left(J\left(\Xi_{d^{\prime}}\right)\right)
\end{array}\right.
$$

The selected parameters contain the main features of the signal received by each sensor (Figure 3). We can regroup these parameters in four categories: the maximal amplitude, the shape, the energy and the difference of viewpoints between these two signals. This set is chosen among the 113 initial features extracted from the received 
signal, its envelope and its fast Fourier transformed (FFT) shown in Figure 2. The initial set of parameters component the maximum amplitude, time of flight (TOF), energy, length of the envelope and some Fourier transform coefficients. Because the device is composed of two sensors we also extract the differences between amplitudes, lengths and distances.
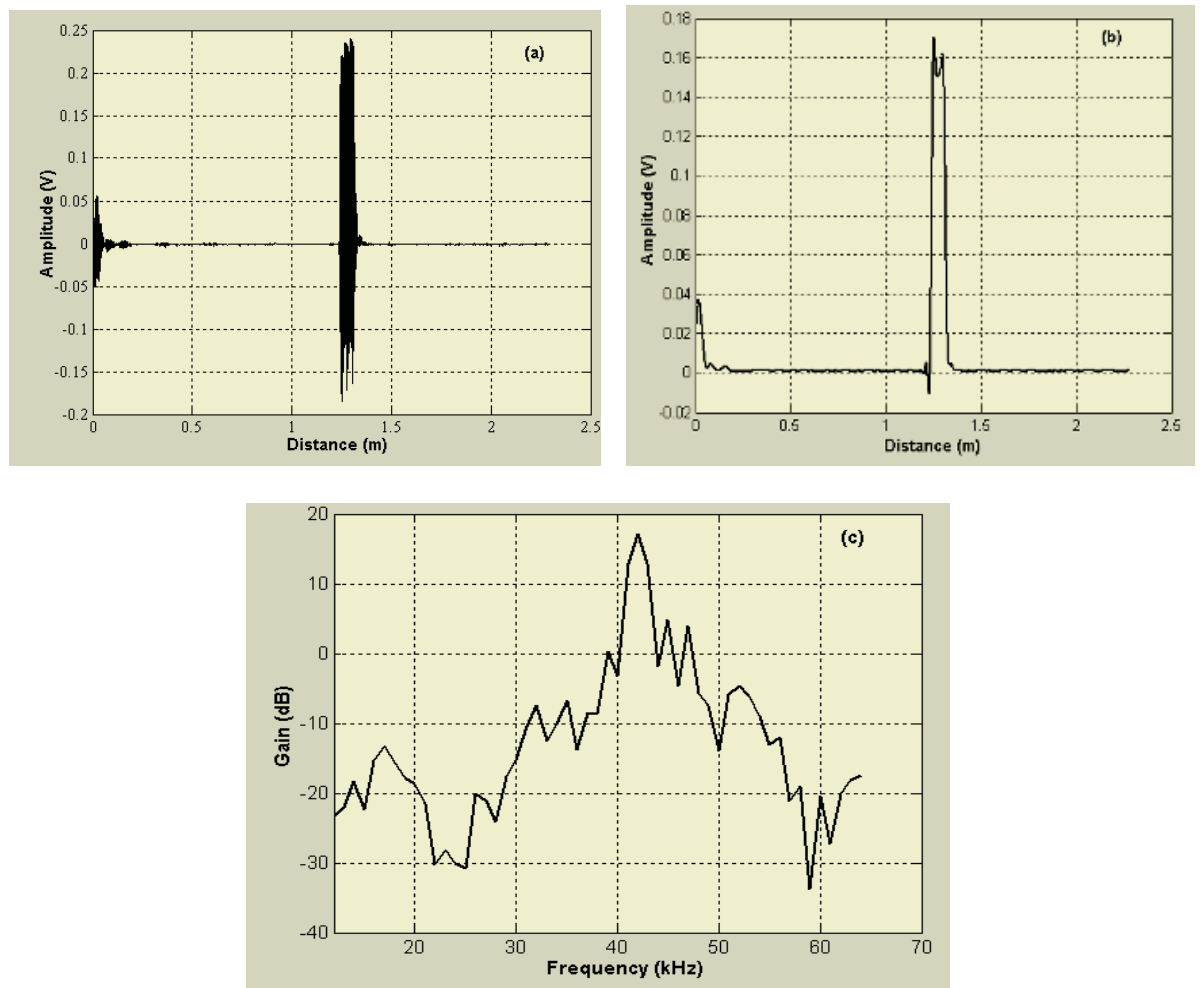

Fig. 2. Feature extraction : (a) real received signal, (b) the envelope of the echo and (c) fast Fourier transformed of the received signal.

\section{Neural Network Classifier}

We compare the classification rate of two supervised networks: Global Neural Network and Specialized Neural Networks. These networks used are a multilayer perceptron with the gradient back-propagation algorithm.

\subsection{Global Neural Network (GNN)}

The network employed has one hidden, one input and one output layer. The hidden layer comprises 18 neurons. This number is determined by the heuristic method, which consists to compare the generalization error for several networks architecture, 
we vary the hidden layer sizes and the input neurons. The number of output layer neurons is equal to the number of classes.

The output is represented by a posteriori probability $\left(\mathrm{P}_{\mathrm{r}}\right)$ of neural classification evaluated by a Softmax function (Equation 1) [14]:

$$
\operatorname{Pr}(k / x)=\frac{\exp \left(y_{k}\right)}{\sum_{j}^{K} \exp \left(y_{j}\right)}
$$

$y_{j}$ is the $\mathrm{j}$ output of the neural classifier,

$K$ is a number of classes.

The maximal probability gives the membership class of example. The classifier is conceived after two phases: learning and generalization using ultrasonic data set.

\subsection{Specialized Neural Networks (SNN)}

This network is composed of several global neural networks (Figure 4). The number of networks is equal to the number of classes. Each network is specialized to recognize one class. The number of output layer neurons is equal to 2 , the class to differentiate and the class "Others" which include all the other classes. Each network has a different architecture that we determine with the heuristic method.

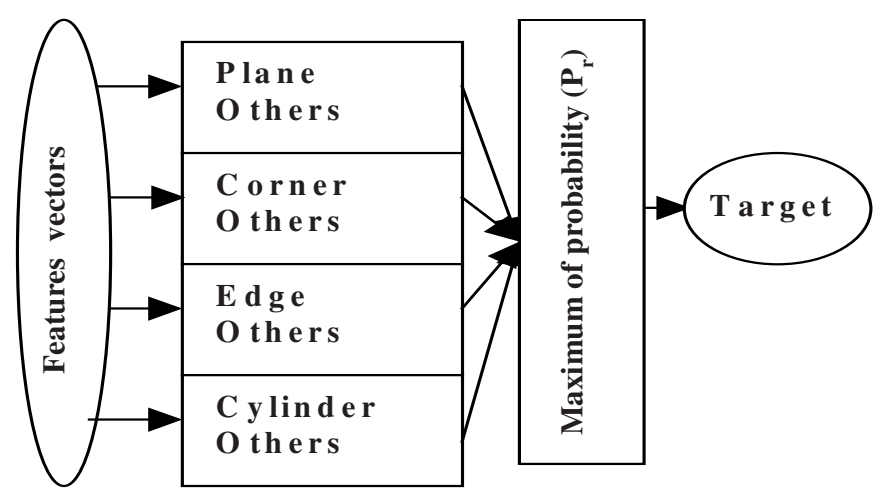

Fig. 3. Specialized Neural Networks architecture.

\section{Results}

\subsection{Data Base}

Two bases of measures have been done, one for the training and another for the generalization. Each one is composed of the four objects, plane, corner, cylinder and edge, placed at various distances from the sonar. The number of examples depends on the dimension of object. For the plane, we use two planes of $16 \mathrm{~cm}$ and $122 \mathrm{~cm}$ width, 
the corner $25 \mathrm{~cm}$ and $48 \mathrm{~cm}$ width, the edge $20 \mathrm{~cm}$ and $43 \mathrm{~cm}$ width and for the cylinder, we use three with different diameter: $16 \mathrm{~mm}, 4 \mathrm{~cm}$ and $20 \mathrm{~cm}$.

The range varies between $0.20 \mathrm{~m}$ to $2.30 \mathrm{~m}$, with an increment of $20 \mathrm{~cm}$. The orientation of the sensor varies between $\pm 20^{\circ}$ with an increment of $5^{\circ}$.

\subsection{Determination of Discriminating Parameter}

Choosing the feature vector dimension is usually a compromise between classification performance and computational time. We compare the FSS and BSS methods, according to the given rate classification by quadratic discriminant analysis (QDA). This method is based on the normal distribution:

$$
f_{k}(X)=(2 \pi)^{-p / 2}\left|\Sigma_{k}\right|^{-1 / 2} \mathrm{e}^{-\frac{1}{2}\left(X-\mu_{k}\right)^{T} \Sigma_{k}^{-1}\left(X-\mu_{k}\right)}
$$

Where $\mu_{\mathrm{k}}$ and $\Sigma_{\mathrm{k}}$ are the class $\mathrm{k}(1 \leq \mathrm{k} \leq \mathrm{K})$ population mean vector and covariance matrix.

\section{Entry Vector of GNN}

We determine the features used like entry vector of GNN with different methods (FSS, BSS and BAB). After comparison between FSS and BSS, we find that FSS gives the best result in term of classification until 30 parameters. We apply the BAB method to refine the final set. We could not use them for more than 30 parameters because of the importance of the computation time.

Table 1. Features selection.

\begin{tabular}{|c|c|c|c|c|}
\cline { 2 - 5 } \multicolumn{1}{c|}{} & \multicolumn{2}{c|}{ FSS } & \multicolumn{2}{c|}{ BAB } \\
\hline Parameters number & 113 & 60 & 30 & $\mathbf{5}$ \\
\hline Mean rate of classification & 94 & 90 & 80 & $\mathbf{7 6}$ \\
\hline
\end{tabular}

The table 1 shows the mean rate of object classification with different number of parameter using QDA. We reduce the dimension of patterns to 5 parameters that are:

- Length of receiver echo, TOF of receiver (R), Difference between the two amplitudes, Two Fourier transform coefficients that correspond to resonance frequency : $50 \mathrm{kHz}$ of transmitter/receiver (T/R) and of receiver $(\mathrm{R})$.

\section{Entry Vector of SNN}

The same methods are applied to obtain the entry vectors of each SNN network.

The SNN structure is illustrated in Table 2, where each target has a different number of features, selected from the initial features set (see section 4) with the different selection feature methods (FSS, BSS and BAB). We choose the features number that correspond to the best rate classification. 
Table 2. SNN structures.

\begin{tabular}{|l|l|l|}
\cline { 2 - 3 } \multicolumn{1}{c|}{} & Size of entry vector & $\begin{array}{l}\text { Classification } \\
\text { rate }(\boldsymbol{\%})\end{array}$ \\
\hline Plane & 7 & 96 \\
\hline Corner & 5 & 92 \\
\hline Edge & 10 & 91 \\
\hline Cylinder & 7 & 95 \\
\hline
\end{tabular}

\subsection{Classification}

The GNN has one hidden layer which comprises 18 neurons. The number of output layer neurons is 4 . The number of input layer neurons is equal to 5 illustrated in ( $\S$ 6.2). The SNN has a different number of hidden layer neurons for each object shown in Table 3. The number of output layer neurons is equal to 2 .

Table 3. Hidden layer neurons.

\begin{tabular}{|l|l|}
\cline { 2 - 2 } \multicolumn{1}{c|}{} & $\begin{array}{l}\text { Hidden layer } \\
\text { neurons }\end{array}$ \\
\hline Plane & 17 \\
\hline Corner & 10 \\
\hline Edge & 13 \\
\hline Cylinder & 15 \\
\hline
\end{tabular}

Table 4 shows the rate of good classification in generalization given by the two methods (GNN, SNN) with the different objects (plane, corner, edge and cylinder).

Table 4. Rate of good classification.

\begin{tabular}{|l|l|l|}
\cline { 2 - 3 } \multicolumn{1}{c|}{} & GNN & SNN \\
\hline Plane & $71 \%$ & $99 \%$ \\
\hline Corner & $73 \%$ & $94 \%$ \\
\hline Edge & $56 \%$ & $96 \%$ \\
\hline Cylinder & $60 \%$ & $83 \%$ \\
\hline
\end{tabular}

The Specialized Neural Networks method (SNN) offers a best rate of classification for all the simple targets (plane, corner, edge and cylinder). This method gives better results because each network is specialized for one class and the estimation of the separation surface between two classes (target and others) is simpler than for the four classes.

\subsection{Classification of Robot Environment}

We tested SNN performances on a robot environment composed of simple targets set (plane, corner and cylinder) with different dimensions. There are three cylinders with different diameters $(20 \mathrm{~cm}, 4 \mathrm{~cm}$ and $2 \mathrm{~cm})$. 
After scanning the environment, the aim is to recognize the targets encountered by the sonar system. We classified the two echoes viewed on the sensor line of sight. We added an ambiguity reject class to the networks output in order to reduce the classification error. We reject an example in ambiguity when probabilities (given by equation 4) of the four networks outputs are equal. The result of classification is superposed on the environment and is shown in the Figure 4.

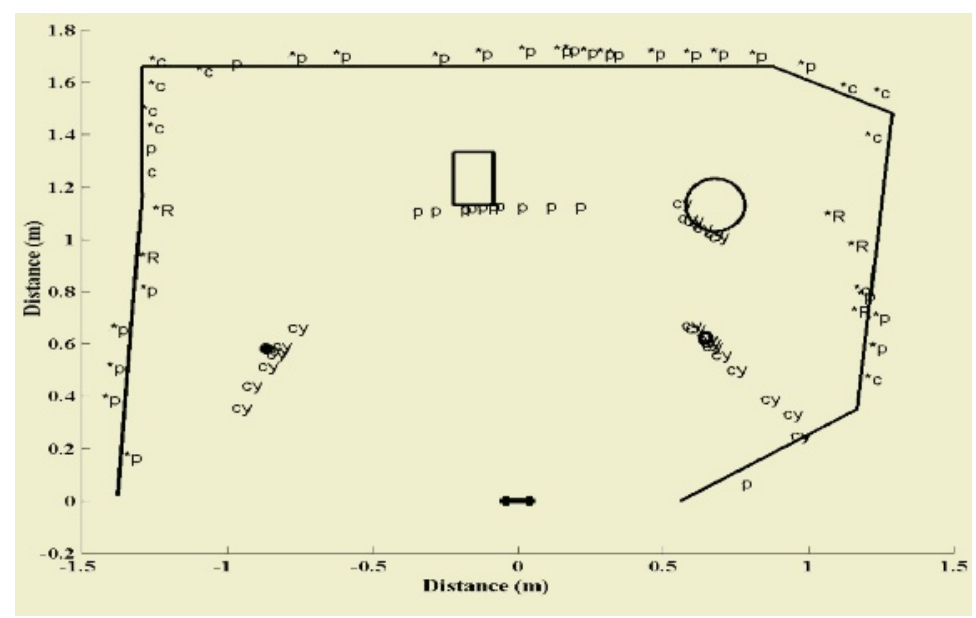

Fig. 4. Classification of a robot environment. Classification are represented by letters: $\mathrm{c}=$ corner, $\mathrm{p}=$ plane, $\mathrm{cy}=\mathrm{cylinder},{ }^{*} \mathrm{p}$ : second plane (second echo encountered on the sensor line of sight) and $\mathrm{R}=$ rejected measure.

The expanded recognition areas are owed to the aperture of the emission signal (plane and cylinder). The error of classification is specially due to the occultation of some objects by others. In this case, the energy reflected by the object occulted is lower than the energy reflected by the first object located in front of the sonar. The classification error of the environment is 3.7\%. The result showed in figure 6 verifies the performance of the SNN method to recognize the simple targets.

\section{Conclusion}

Neural networks are employed to process real sonar data after trained to learn identifying relations for the target primitives. In this study, The SNN method is compared to the GNN method to classify 2D indoor environment based on ultrasonic measures. The best result is obtained with the SNN method. The information included in the echo is sufficient to classify the environment in four classes (edge, corner, plane and cylinder) with good performances. The results confirm the value of sonar as a sensor for object recognition and suggested wider use of neural networks as robust pattern classifiers in sensor-based robotics. For future works it is important to consider the unsupervised learning algorithms to make the classification process more robust to changes in environmental conditions. 


\section{References}

1. Borenstein, J., Everett, H., and Feng, L. Navigating mobile robots. A.K. Peters, Wellesley, MA. (1996).

2. Leonard, J. J.; and Durrant-Whyte, H. F. Directed sonar sensing for mobile robot navigation. The Kluwer international series in engineering and computer science. Kluwer Academic Publishers, Boston, (1992).

3. Barshan, B.;Kuc, R. Differentiating sonar reflections from corners and planes by employing an intelligent sensor. IEEE Trans. Pattern Anal. (1990).

4. Barshan, B., Ayrulu,B., Utete, S.W. Neural Network-Based Target Differentiation Using Sonar for Robotics Applications. IEEE Trans, Robotics and Automation, (2000), 435-442.

5. Bozma, Ö. ; Kuc, R. Building a sonar map in a specular environment using a single mobile sensor. IEEE Trans. Pattern Anal and Machine Intell, (1991).

6. Kuc, R. Three-dimensional tracking using qualitative bionic sonar. Robotics Autonomous Systems, (1993). 213-219.

7. Borenstein, J.; and Koren, Y Obstacle avoidance with ultrasonic sensors. IEEE Journal of Robotics and Automation, Vol. 4, N.2, (1988). 213-218.

8. Roiblat, H. L., Au. W. W. L., Nachtigall. P. E., Shizumuru. R, and Moons. G., Sonar recognition of targets embedded in sediment, Neural Network, G. B. (1995) 1263-1273,

9. Carpenter et al. Fuzzy ARTMAP: A neural network architecture for incremental supervised learning of analog multidimensional maps. IEEE Transactions on Neural Networks, 3(5), (1992), 698-712

10. Willson, Radar classification using a neural network, Proc. SPIE, optical Engineering and photonics in aerospace sensing, (1990). 200-210

11. Peremans, H., Audenaert, K., Van Campenhout, J. M.,. A high resolution sensor based en tri-aural perception. IEEE Trans on Robotic and Automation, CA, (1993)

12. Jain, K., Duin, P. W, Mao, J. Statistical pattern recognition : A review. IEEE Trans. Pattern Anal and Machine Intell, (2000).

13. Ait Oufroukh, N.; Colle, E. Pattern Recognition with Ultrasonic Sensor using Classification Methods. $1^{\text {st }}$ Hybrid intelligent system (HIS), Chili, (2002), 673-680,

14. Bishop, C. Neural Networks for Pattern Recognition. Clarendon Press, Oxford, (1995). 